\title{
Extranodal Rosai-Dorfman of the Pancreas Presents with Bilateral Granulomatous Anterior Uveitis
}

\author{
A'sha Brown $^{a}$ Sara V. Branson ${ }^{b}$ Ghazala A. Datoo O'Keefe ${ }^{c}$ \\ ${ }^{a}$ Emory University School of Medicine, Emory Eye Center, Medical Retinal and Uveitis Fellow, Atlanta, GA, USA; \\ ${ }^{b}$ Emory University School of Medicine, Emory Eye Center, Resident, Atlanta, GA, USA; ' $E$ Emory University School of \\ Medicine, Assistant Professor of Ophthalmology, Section of Vitreoretinal Surgery and Diseases, Section of Uveitis \\ and Vasculitis, Atlanta, GA, USA
}

\section{Established Facts}

- Rosai-Dorfman Disease (RDD) is known to affect many organ systems. Ocular disease only occurs in around $10 \%$ of cases.

- Uveitis is a very rare ocular manifestation of RDD.

\section{Novel Insights}

- RDD should be considered in the differential of a patient with bilateral anterior uveitis.

- Systemic workup of ocular findings can reveal other systemic findings of RDD.

\section{Keywords}

Uveitis · Rosai-dorfman disease · Sinus histiocytosis with massive lymphadenopathy · Pancreatic mass

\footnotetext{
Abstract

Background/Aims: To report a case of a 65-year-old female who presented with bilateral anterior granulomatous uveitis, which led to the diagnosis of Rosai-Dorfman Disease (RDD) and the discovery of an associated pancreatic mass. Methods: This is a retrospective case report of a single patient. Results: A 65-year-old African American Female re-
}

ceived a full workup for bilateral anterior granulomatous uveitis and was found to have a pancreatic mass on imaging. Biopsy of this mass was diagnostic for RDD, a rare proliferative histiocytic disorder, which classically presents with painless cervical lymphadenopathy, leukocytosis, and hypergammaglobulinemia. Conclusion: Although rare, ocular involvement, including eyelid and orbital masses, and anterior uveitis, can herald the development of other systemic manifestations or RDD and can aid in the diagnosis of this systemic disease.

\section{KARGER}

E-Mail karger@karger.com

www.karger.com/oop
(C) 2018 S. Karger AG, Basel Ghazala A. Datoo O'Keefe, MD

Section of Vitreoretinal Surgery and Diseases

Section of Uveitis and Vasculitis Emory University School of Medicine

Atlanta, GA 30322 (USA)

E-Mail g.d.okeefe@emory.edu 


\section{Introduction}

Rosai-Dorfman Disease (RDD), also known as sinus histiocytosis with massive lymphadenopathy, is a rare benign proliferative histiocytic disorder for which the pathogenesis is not well elucidated. It typically presents within the first 2 decades of life with self-limited painless cervical lymphadenopathy and is commonly accompanied by leukocytosis, hypergammaglobulinemia, and an elevated erythrocyte sedimentation rate. Extranodal manifestations have been noted in up to $28-43 \%$ of cases, including the involvement of the skin, gastrointestinal system, visceral organs, respiratory tract, and nervous system $[1,2]$. Ocular involvement is rare, and usually involves lymphoproliferative tissue in the orbit or eyelids, with few reports of uveitis. Although ocular findings do not always herald systemic involvement, recognition of ocular disease can often lead to the diagnosis of underlying systemic involvement. We present the first case of RDD with bilateral anterior granulomatous uveitis associated with a pancreatic mass.

\section{Presentation of Case}

A 65-year-old African-American female presented with bilateral eye pain, redness, and photophobia. Pertinent past medical history includes a history of skin rash of the extremities, which consisted of numerous annular violaceous plaques with biopsy suspicious for granulomatous dermatitis. On initial exam, VA was $20 / 15$ both eyes (OU) with trace anterior chamber cell OU. She was treated with prednisolone acetate $1 \%$ drops with a taper. Six months after the discontinuation of the steroids, she experienced recurrence of symptoms and was seen in uveitis clinic. At that time, she had conjunctival injection, increased anterior chamber cell to $2+$, and keratic precipitates arranged circumferentially around the cornea in both eyes (Fig. 1a, b). The fundus exam was unremarkable. A complete uveitis workup, including serologic studies (Quantiferon gold, human immunodeficiency virus, dsDNA (anti-double stranded DNA), ANA (antinuclear antibody), Treponemal antibody, ANCA (antineutrophil cytoplasmic antibody), RF (rheumatoid factor)), was negative. She had an elevated ESR (erythrocyte sedimentation rate) (34) and CRP (C-reactive protein) (22.6). Computed tomography of the chest was performed to rule out sarcoidosis and showed mediastinal lymphadenopathy without hilar adenopathy and a mass of the pancreatic tail $(1.8 \times$ $1.9 \mathrm{~cm}$ ) suspicious for intraductal papillary mucinous neoplasm or pancreatic adenocarcinoma. A follow-up MRI study was done and was also suspicious for malignancy (Fig. 1c).

The patient was referred to gastroenterology and underwent a laparoscopic distal pancreatectomy/splenectomy with resection of a well-circumscribed pancreatic mass (Fig. 1d). The histology showed predominately lymphocytes with emperipolesis and immunostains were positive for S100 and CD163 at which time the patient was diagnosed with RDD (Fig. 2).
Treatment was continued with topical prednisolone acetate $1 \%$, which led to the resolution of uveitis and stable visual acuity at 7 months after initial presentation.

\section{Discussion}

Ocular disease in $\mathrm{RDD}$ is rare, and is thought to occur in around $10 \%$ of cases, most commonly presenting as an orbital or epibulbar mass [2-5]. Uveitis is rarely associated with RDD, and only 13 cases have been reported in the English literature in the past 2 decades (Table 1). Patients most commonly present as chronic recurrent bilateral granulomatous uveitis and overall have a good visual prognosis [6]. Treatment usually entails topical and oral steroids, but radiation, chemotherapy, and surgical excision is used in cases with severe systemic disease. Poor visual outcomes in the literature have been associated with the development of complications including uveitic glaucoma, cystoid macular edema with serous retinal detachment, and panuveitis $[4,7,8]$.

Investigative clinical work up for nodal RDD includes CT head and neck, chest, abdomen, and pelvis with excisional lymph node or extranodal tissue biopsy and extensive infectious workup. Patients with uveitis should also undergo full uveitis workup to rule out other etiologies. Histology is required for definitive diagnosis and shows a histiocytic proliferation that demonstrates emperipolesis and is S-100 positive, CD68 positive, and CD1a negative [1]. It has been postulated that uveitis occurs through the histiocytic infiltration of the uvea, as has been shown in an enucleated eye of a patient with orbital RDD. However, biopsy of uveal tissue is not commonly performed and little evidence is available to support this hypothesis [2]. Close histological examination is important to differentiate RDD from other lymphocytic diseases including histiocytosis X, histiocytic lymphoma, orbital pseudotumor, Hodgkin's lymphoma, and monocytic leukemia. Epibulbar lesions in RDD can mimic both lymphoma and scleritis $[4,9,10]$, necessitating biopsy if the lesion did not improve with treatment. Although the etiology is unknown, RDD is thought to be a reactive process and has been associated with Epstein-Barr virus [10], varicella zoster [11], human immunodeficiency virus [12], and other infectious etiologies such as tuberculosis $[7,13]$. There is also notable association with autoimmune conditions such as hypothyroidism $[4,7,13]$. Laboratory studies are inconsistently reported in literature and more work is necessary to elucidate the cause of this disease [1]. 

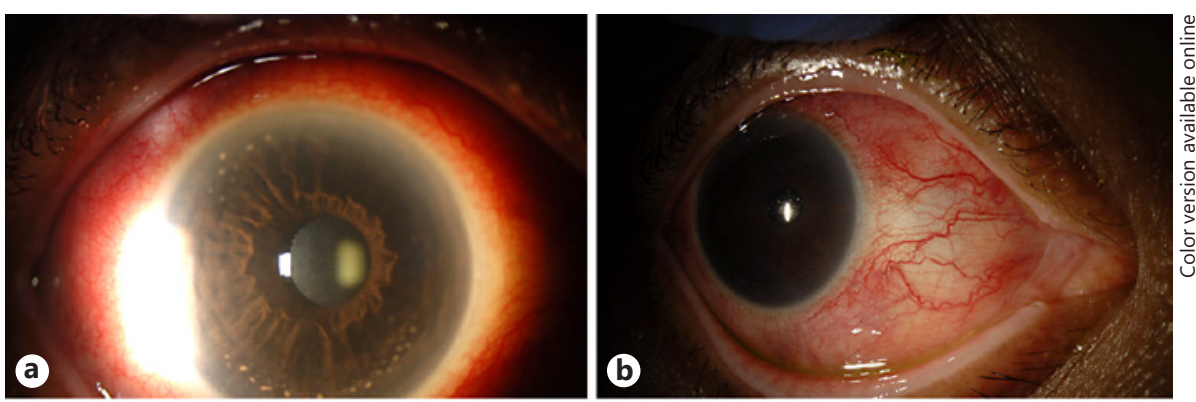

Fig. 1. Clinical photographs and imaging from case. a, b Slit lamp photographs of the patient at presentation showing diffuse circumferential keratic precipitates (a) and diffuse conjunctival injection with ciliary flush (b). c MRI abdomen, T-1 sequence showing hypoenhancing lesion of the pancreatic tail, suspicious for pancreatic adenocarcinoma. d Gross specimen of resected pancreatic mass measuring $2.1 \times 1.7 \times$ $1.1 \mathrm{~cm}$.
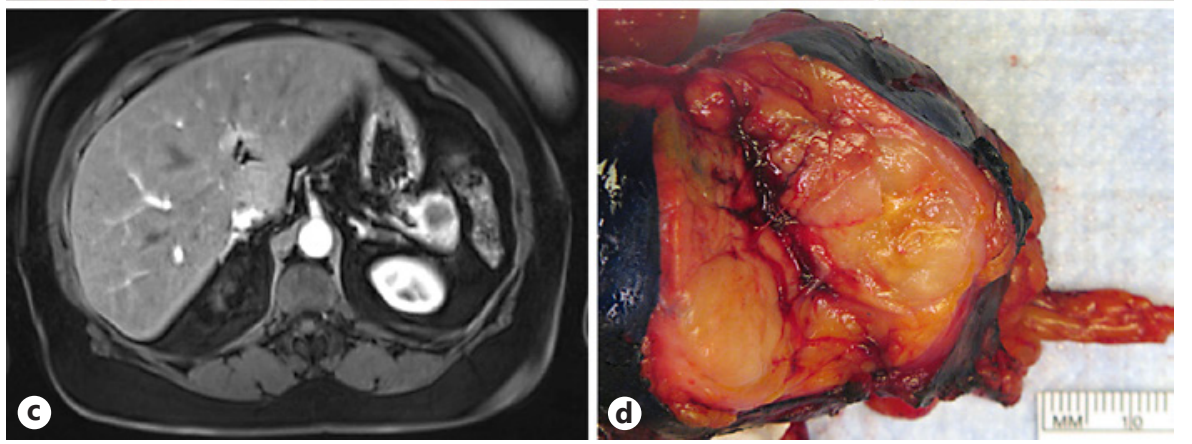

Fig. 2. Histology with immunohistochemistry. a Histology shows abundant histiocytes with foamy eosinophilic cytoplasm and interspersed chronic inflammatory cells. b Demonstration of emperipolesis, the characteristic feature of RDD in which histiocytes contain phagocytosed lymphocytes. c, d Immunohistochemical stains positive for CD163 (c) and S100 (d), consistent with RDD. All photos hematoxylin and eosin; 20× magnification.

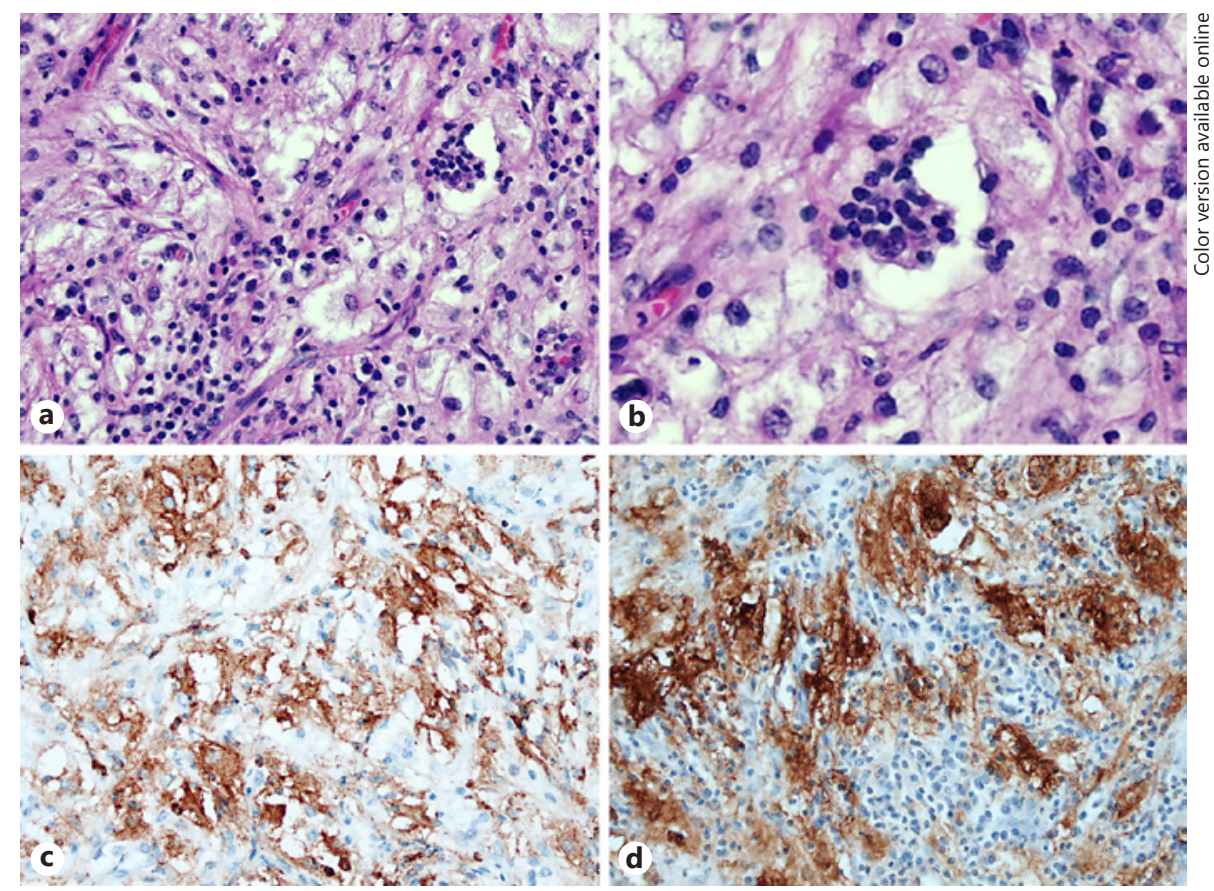

RDD demonstrates high clinical variability with regard to the severity and duration of disease but is usually self-limited with an overall good prognosis. There does not appear to be a well-defined correlation of ocular involvement with the clinical phenotype or prognosis of this disease. Foucar et al. [2] suggests that patients with ophthalmic disease may be more likely to have an additional site of extranodal involvement (54\%) versus those without ophthalmic disease (28\%), and patients with eye findings may have a higher rate of nasal sinus involve- 
Table 1. Cases of RDD with uveitis

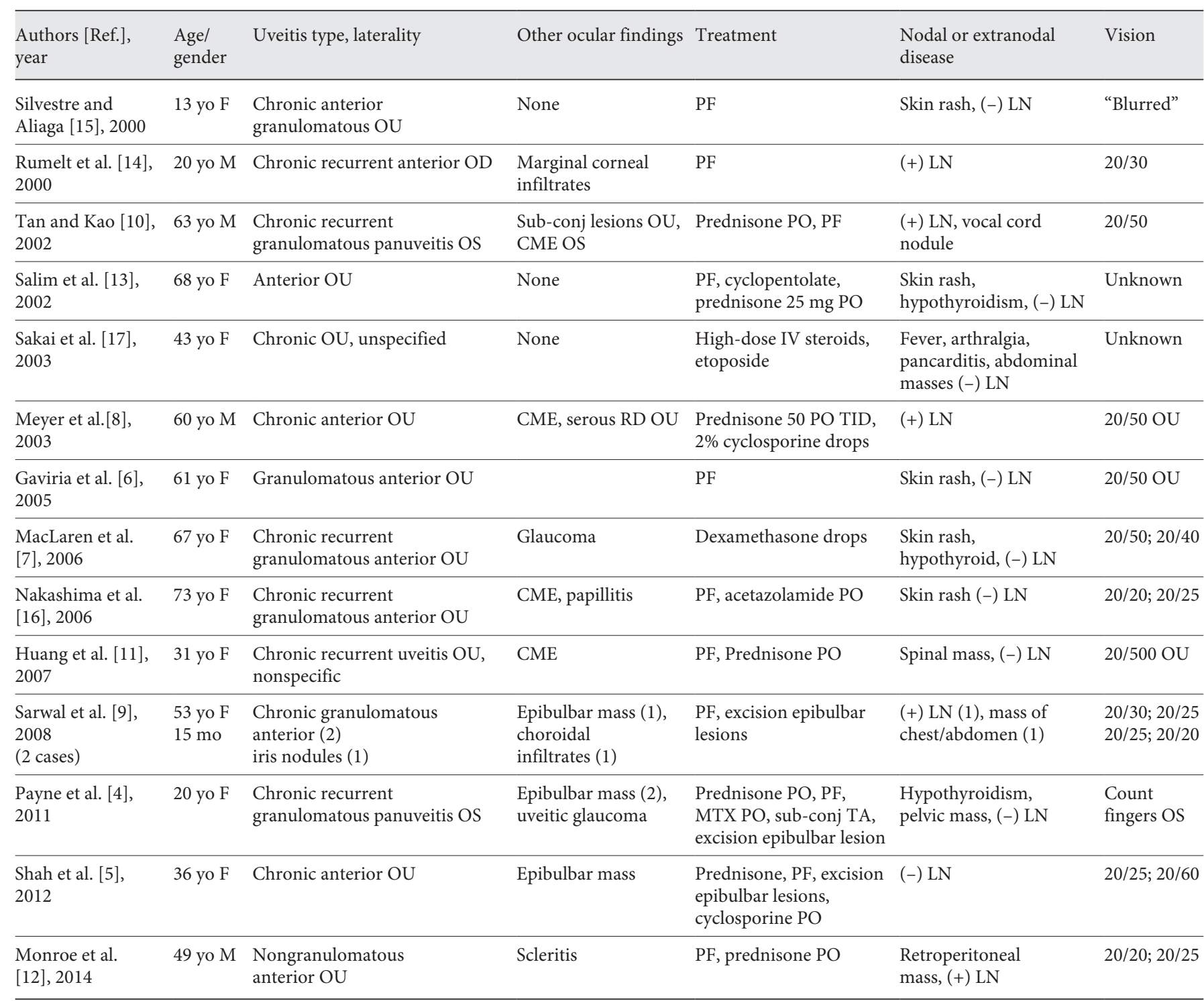

OU, both eyes; OD, right; OS, left; PF, pred forte (prednisolone acetate 1\%); RDD, Rosai-Dorfman disease; CME, cystoid macular edema; RD, retinal detachment; VA, visual acuity; (+/-) LN, with or without lymphadenopathy; TA, triamcinolone acetonide; yo, years old; F, female; M, male.

ment. In review of cases of uveitis with RDD from the past 2 decades, only 4 of the 13 cases had nodal disease [8-10, 14]. All cases except $2[8,14]$ had extranodal manifestations in addition to ocular findings. Our patient presented with cutaneous involvement, which is cited as the most common extranodal presentation $[6,7,13,15,16]$. In this case, the patient initially presented with a rash for which the biopsy was non-diagnostic. Performing the workup for uveitis resulted in the incidental discovery of the pancreatic mass and mediastinal lymphadenopathy, which led to the diagnosis of RDD. Uveitis has been shown to precede systemic symptoms by months to years in some cases $[4,6,7,11,13,17]$. However, in most cases, a thorough workup including imaging or biopsy was not performed until other systemic manifestations presented. Huang et al. [11] describes a case of spinal cord compression from an epidural mass associated with RDD which was preceded by 6 months of chronic relapsing uveitis necessitating urgent thoracic laminectomy. Another notable feature is that 6 of the 13 cases reviewed in literature 
as well as this case featured a recurrent pattern of uveitis, suggesting that this feature should raise suspicion for $\operatorname{RDD}[4,7,10,11,14,16]$.

This case was an atypical presentation of RDD without cervical lymphadenopathy, fever, or other typical RDD signs aside from a nonspecific rash. The pancreatic mass was an incidental finding, but led to the prompt resection of the mass to rule out malignancy. The ophthalmologist should suspect RDD in a patient with bilateral anterior granulomatous uveitis and systemic symptoms such as a rash or lymphadenopathy, and perform the full workup to identify possible systemic complications.

\section{Statement of Ethics}

The authors have no ethical conflicts to disclose.

\section{Disclosure Statement}

The authors have no conflict of interest to declare.

\section{References}

1 Cai Y, Shi Z, Bai Y. Review of Rosai-Dorfman Disease: New Insights into the Pathogenesis of This Rare Disorder. Acta Haematol. 2017; 138(1):14-23.

2 Foucar E, Rosai J, Dorfman RF. The ophthalmologic manifestations of sinus histiocytosis with massive lymphadenopathy. Am J Ophthalmol. 1979 Mar;87(3):354-67.

3 Rosai J, Dorfman RF. Sinus histiocytosis with massive lymphadenopathy: a pseudolymphomatous benign disorder. Analysis of 34 cases. Cancer. 1972 Nov;30(5):1174-88.

4 Payne JF, Srivastava SK, Wells JR, Grossniklaus HE. Rosai-Dorfman disease simulating nodular scleritis and panuveitis. Arch Ophthalmol. 2011 Apr;129(4):518-20.

5 Shah A, Bielory L, Mirani N, Tu Y, Chu DS. Epibulbar rosai-dorfman disease: novel manifestation and treatment. Arch Ophthalmol. 2012 Sep;130(9):1218-20.

6 Gaviria JG, Johnson DA, Kinney MC, Proffer LH, Losi-Sasaki JM, Kraus EW. Bilateral anterior granulomatous uveitis associated with cutaneous Rosai-Dorfman disease. Graefes Arch Clin Exp Ophthalmol. 2005 Mar;243(3): 281-4.
7 MacLaren RE, Hundal KS, Trittibach P, Bloom PA. Uveitic glaucoma and Rosai-Dorfman disease (sinus histiocytosis). Ocul Immunol Inflamm. 2006 Oct;14(5):305-7.

8 Meyer CH, Sel S, Hörle S, Burg J, Kroll P. Rosai-Dorfman disease with bilateral serous retinal detachment. Arch Ophthalmol. 2003 May;121(5):733-5.

9 Sarwal R, Tu E, Mendelblatt FI, Sugar J, Gross SA, Pulido JS, et al. Atypical ocular presentations of Rosai-Dorfman disease. Ocul Immunol Inflamm. 2008 Jan-Feb;16(1):9-15.

10 Tan HY, Kao LY. Rosai-Dorfman disease manifesting as relapsing uveitis and subconjunctival masses. Chang Gung Med J. 2002 Sep;25(9):621-5.

11 Huang YC, Tan HY, Jung SM, Chuang WY, Chuang CC, Hsu PW, et al. Spinal epidural Rosai-Dorfman disease preceding by relapsing uveitis: a case report with literature review. Spinal Cord. 2007 Sep;45(9):641-4.
12 Monroe AK, Burkholder BM, Butler NJ, Duffield AS. Sclerouveitis associated with RosaiDorfman disease in an HIV-infected patient. Ocul Immunol Inflamm. 2014 Jun;22(3):2368.

13 Salim A, Williamson M, Barker F, Hughes J. Steroid responsive cutaneous Rosai-Dorfman disease associated with uveitis and hypothyroidism. Clin Exp Dermatol. 2002 Jun;27(4): 277-9.

14 Rumelt S, Cohen I, Rehany U. Marginal corneal infiltrates: a possible new manifestation of sinus histiocytosis with massive lymphadenopathy. Cornea. 2000 Nov;19(6):857-8.

15 Silvestre JF, Aliaga A. Cutaneous sinus histiocytosis and chronic uveitis. Pediatr Dermatol. 2000 Sep-Oct;17(5):377-80.

16 Nakashima M, Matsui Y, Kobayashi S. Relapsing uveitis in association with presumed sinus histiocytosis. Jpn J Ophthalmol. 2006 Sep-Oct;50(5):484-6.

17 Sakai Y, Atsumi T, Itoh T, Koike T. Uveitis, pancarditis, haemophagocytosis, and abdominal masses. Lancet. 2003 Mar;361(9360):834 